\title{
El tratamiento museográfico del arte rupestre en España
}

\author{
Miryam Galaz Villasante * \\ maría Dolores Moneva Montero *
}

\section{INTRODUCCIÓN}

El arte rupestre prehistórico constituye una de las categorias más signficativas y originales del Patrimonio cultural español y es, sin lugar a dudas, una de las que más interés despierta entre el público no especializado ${ }^{1}$. Sin embargo, la proyección social de este rico conjunto de bienes presenta graves dificultades debido a toda una serie de factores, entre los que sobresalen su carácter inmueble, especiales condicionamientos geográficos y extrema fragilidad.

Tradicionalmente el Patrimonio ha encontrado en los museos uno de los vehículos más adecuados de contacto con la sociedad. Esta alternativa, que es válida para los objetos muebles (desde piezas de mobiliario y pinturas a documentos), choca con graves dificultades en el caso de los bienes inmuebles (obras de arquitectura, ingenieria, escultura colosal y conjuntos arqueológicos), si bien es cierto que estos últimos también se exhiben, con mayor o menor fortuna, en las salas de muchos museos.

El objetivo de este trabajo, fruto de un doble interés por el mundo de la museología y de la arqueología, es ofrecer una valoración de la actual situación del arte rupestre en los museos españoles. Esta investigación

* Becarias de Investigación de la Comunidad de Madrid en el Departamento de Prehistoria y Arqueologia de la U.A.M.

1 Su inclusión como bien patrimonial está definida en el título V, artículo 40.2 de la Ley 16/85 de Patrimonio Histórico Español. En 1986 se crea la Junta Superior de Arte Rupestre, institución consultiva de la Administración del Estado, adscrita a la Dirección General de Bellas Artes y Archivos, con el fin de supervisar todas las actuaciones relativas a esta particular categoria de bienes. Asimismo, la Carta del Restauro de Roma (1987) se hace eco de la necesidad de proteger estas singulares manifestaciones (Anexo B, capítulo 6). 
no hubiera sido posible sin la inestimable colaboración de los responsables de dichas instituciones, a través de las respuestas y observaciones a un cuestionario elaborado por las autoras en relación al tema objeto de estudio (Anexos 1 y 2 ).

Dos son los principales problemas a los que se enfrenta una investigación de esta naturaleza: la escasez de referencias bibliográficas existentes ${ }^{2} y$, como se verá, la poca valoración del arte rupestre como objeto museográfico por parte de muchos de los profesionales consultados.

\section{EL TRATAMIENTO MUSEOGRÁFICO DEL ARTE RUPESTRE}

La museización del arte rupestre se ha realizado tradicionalmente de dos maneras:

a) Exposición uin situm: Es decir, transformando las cuevas, abrigos o peñones decorados en "museos" mediante intervenciones, a veces muy drásticas, que suelen consistir en la adecuación de los enclaves para su visita (accesos, aparcamientos, taquillas, iluminación), la contratación de guías (de carácter permanente o semipermanente) y, más puntualmente, la instalación de pequeños museos de yacimiento (como sucede en dos cuevas paleolíticas de la cornisa cantábrica: Altamira y Tito Bustillo).

b) Exposición en el Museo: Incluyendo el arte rupestre en las salas mediante el recurso a distintos tipos de reproducciones (calcos, dibujos, fotografías), agrupadas según disposiciones museográficas diversas. Algunas entidades todavía conservan piezas originales, fruto de arranques en su mayoría realizados durante las primeras décadas de este siglo, por causas difícilmente justificables. Sin duda los fragmentos del abrigo levantino de Calapatá (Cretas, Teruel), procedentes de la colección de Juan Cabré y comprados por el Museo Arqueológico de Barcelona, constituyen uno de los ejemplos más conocidos ${ }^{3}$.

2 A pesar de las numerosas gestiones realizadas no se han podido localizar dos de las escasas publicaciones referidas al tema, concretamente: CASANOVAS, A., y ALONSO, A. (1989): "Notas acerca de la integración del arte rupestre en el discurso museístico", Boletín de la Asociación Española de Arte Rupestre II, y el congreso Art rupestre et muséologie. Milán (29-31 de octubre de 1982).

3 Poco tiempo después del arranque de Calapatá, sucedido hacia 1905, la Comisión de Investigaciones Paleontológicas y Prehistóricas envió al Museo de Ciencias Naturales de Madrid fragmentos de dos abrigos de Aldeaquemada (Jaén) y de la cueva del Bullón (Villar del Humo, Cuenca), de los que nada se sabe en la actualidad. En el Museo de Cervera (Lérida) se conserva un arquero de la Cova dels Cavalls (Valltorta, Castellón). 
La aportación más interesante de los últimos años ha sido, sin lugar a dudas, la creación de los denominados Parques Culturales, solución válida para muchas categorías de bienes, entre las que se incluyen las manifestaciones artísticas rupestres, y que ya cuenta con una larga experiencia fuera de nuestras fronteras.

Según Baldellou (1990: 151), el parque cultural representa «la vía óptima para que las pinturas rupestres prehistóricas lleguen al público del mejor modo posible: dentro de su ecosistema original, insertas en el entorno ambiental en el que fueron concebidas».

El primer proyecto de parque cultural dedicado al arte rupestre en España fue el del conjunto levantino del barranco de La Valltorta (Castellón), presentado en el Coloquio de Albarracín de 1986 y lamentablemente paralizado en la actualidad. Precisamente en el curso de dicha reunión se propuso la creación de dos nuevos proyectos, ambos en Aragón: el de Albarracín (Teruel) y el del río Vero (Huesca). A ellos se suma el proyecto de la Cueva Pintada de Gáldar (Gran Canaria), incluido en el Plan Nacional de Parques Arqueológicos, promovido por el Ministerio de Cultura, y el de la zona de Valonsadero (Soria), que engloba uno de los más destacados conjuntos de arte esquemático de la Meseta superior. Otro espacio natural protegido con arte rupestre, con testimonios paleolíticos y esquemáticos, es el parque de Ardales (Málaga) ${ }^{4}$.

A pesar de las innegables ventajas del Parque Cultural en las labores de conservación y divulgación del arte rupestre, éste no puede, ni debe, invalidar la tradicional exposición en los Museos. Por una parte, es evidente que no todas las estaciones artísticas son susceptibles de convertirse en parques culturales, por factores de muy diversa indole (científicos, económicos o de salvaguarda 5); por otra parte, muchos de los dispositivos museográficos más modernos y didácticos encuentran en las salas del museo tradicionalmente entendido su marco más idóneo de ejecución (HALL, 1987).

4 Sobre la Valltorta, véase Generallitat Valenciana, 1985; para Albarracín, véase Collado VIllalba, 1992; acerca de Vero, Baldellou, 1989; Gáldar, véase MARTín de GuzMán, 1993, y sobre Valonsadero, véase Gómez BARRera et al. 1989.

Para una revisión crítica de las actuaciones ya acometidas, véase BALldellou et al. 1988 y las Jornadas sobre Parques con Arte Rupestre, 1989.

5 Altamira (Santillana del Mar, Cantabria), una de las estaciones más emblemáticas y visitadas, tuvo que cerrarse en octubre de 1977 por el creciente deterioro antrópico y actualmente dispone de un sistema de visita restringida (HerRero, c. 1983a). 


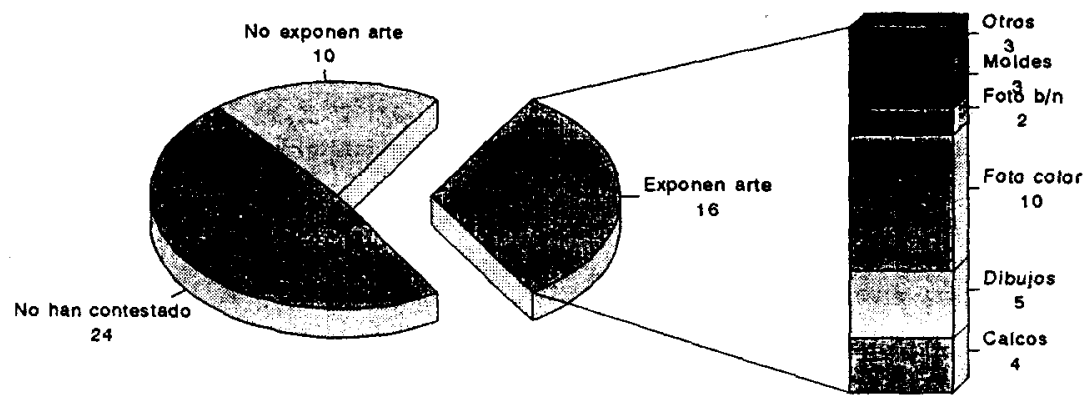

Dispositivos museográficos

Fig. 1. Arte rupestre en los museos españoles: resultados de la encuesta (diciembre 1993-mayo 1994).

\section{SITUACION ACTUAL DEL ARTE RUPESTRE EN LOS MUSEOS ESPAÑOLES}

Con la finalidad de conocer el tratamiento del arte rupestre en los museos españoles, se elaboró un cuestionario en el que figuraban preguntas relativas a su presentación actual en las salas y en el que se recababa la opinión de los especialistas sobre posibles transformaciones en las mismas (Anexo 1).

La investigación se desarrolló entre los meses de diciembre de 1993 y abril de 1994. El número total de instituciones consultadas fue de 50 , de las cuales, a finales de mayo de 1994, habian respondido 26 (52\%), siendo $16(61 \%)$ las que exponian arte rupestre entre sus fondos (Anexo 2) (figura 1).

Del análisis de las respuestas obtenidas en los museos que exponen arte rupestre, se desprenden los siguientes datos:

1. Criterios de elección de estaciones artísticas: Por lo general, los museos tienden a exponer el arte de su propio entorno (provincial o regional), salvo en tres casos: el Museo Arqueológico Nacional, dado su carácter de unacional», y los de Valladolid y Barcelona, que han elegido ejemplos europeos (Dordoña, Francia) y españoles (Altamira y Calapatá 


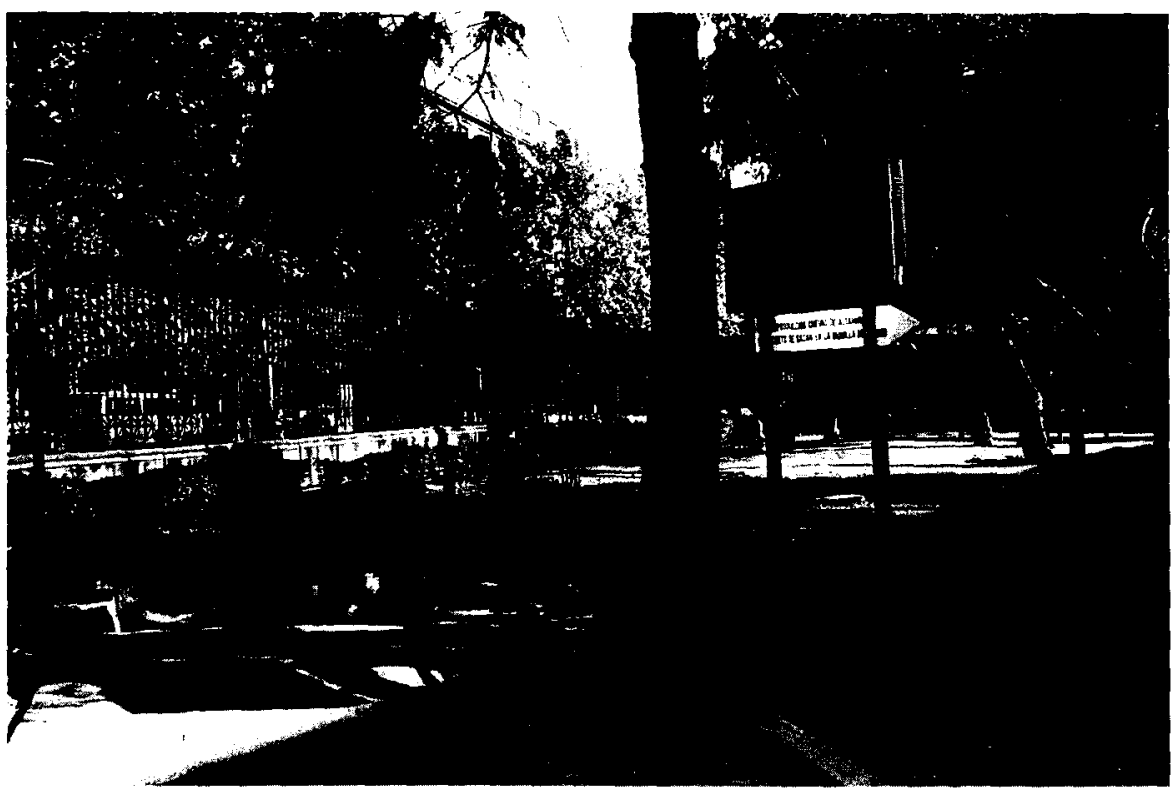

Fig. 2. Entrada al facsímil de Altamira ubicado en los jardines del Museo Arqueológico Nacional.

entre otros), debido, probablemente, a la trascendencia de las manifestaciones que estas estaciones contienen.

2. Localización de los testimonios en el museo: En el Museo Arqueológico Nacional existe una sala específicamente acondicionada para la exposición de arte rupestre: Sala de los Polícromos de Altamira (fig. 2). También el Museo de Pontevedra cuenta con un espacio concreto para el arte rupestre. Las demás instituciones optan por integrar el arte en las salas correspondientes, según criterios cronológicos y como apoyo a la explicación general del período correspondiente.

3. Sistemas de reproducción (fig. 3): Los sistemas más utilizados son la fotografía en color y, en menor medida, el dibujo, el calco y los diferentes tipos de moldes. El recurso a la fotografía en blanco y negro se explica por la antigüedad de buena parte de las instalaciones.

El material procede, en su mayoría, de las campañas de investigación, no habiendo sido específicamente concebido para el museo.

El Museo de Pontevedra dispone de unos treinta moldes de petroglifos de diferentes municipios de la provincia (Campolameiro, Cotobade, Poio, etc.). Los más antiguos proceden de la colección de Sobrino 


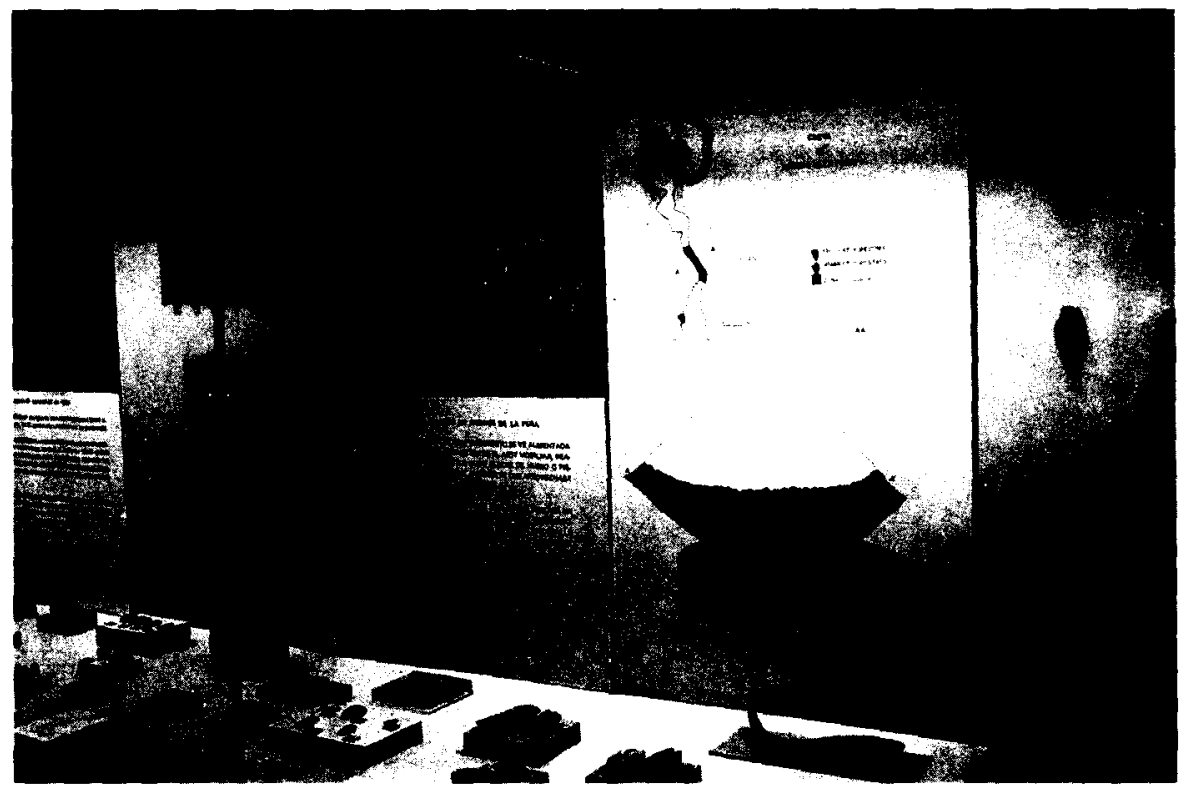

Fig. 3. Integración del arte rupestre en el discurso museístico (Sala de Prehistoria del Museo Arqueológico Nacional).

Buhigas y fueron realizados en el primer cuarto del siglo xx. En los años setenta se incorporaron los de la colección C.G. Borgna (1972) y los de la Diputación Provincial (1974).

En el Museo Arqueológico de Segovia ${ }^{6}$ se conserva el molde del caballo paleolítico de Domingo Garcia (Segovia), obra de Alejandro Gallego Fernández, técnico de laboratorio del Departamento de Prehistoria y Arqueología de la Universidad Autónoma de Madrid, realizado en diciembre de 1991 a instancias de la Dra. Rosario Lucas Pellicer (figs. 4-5). Se trata de un molde directo de latex al que se ha dado la apariencia de la roca original mediante utilización de tierra y restos vegetales recogidos "in situ» 7 . Se trata de uno de los pocos ejemplos conocidos de moldes de grabados, ya que, en la mayoría de los casos, se opta por la reproducción de motivos pintados (Delluc, 1990).

- Actualmente en proceso de remodelación.

7 Formó parte de la Exposición sobre Arte Rupestre en las Comunidades de Castilla y León y Madrid realizada en la Universidad Autónoma de Madrid en enero de 1992 y posteriormente fue entregado al Museo de Segovia. En dicha Universidad también se conserva una copia de este molde. 


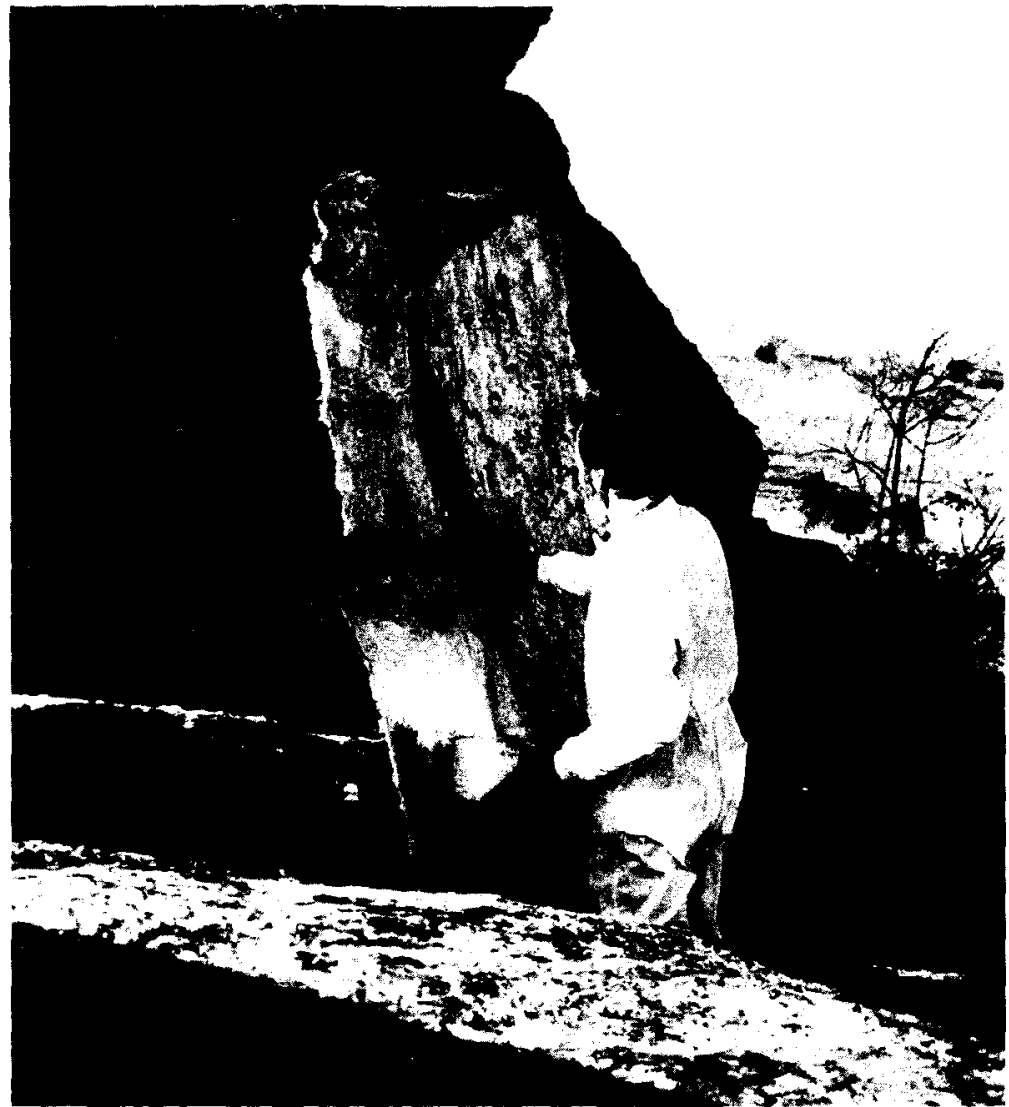

Fig. 4. Proceso de ejecución del molde del grabado del caballo paleolítico de Domingo Garcia, Segovia (fotografia facilitada por Alejandro Gallego Femández).

Uno de los dispositivos más antiguos del Museo Arqueológico de Barcelona es el diorama (molde con calco) de la Cueva de Altamira, que goza de un gran favor por parte del público a pesar del tiempo transcurrido.

También está dedicado a Altamira el facsímil del Museo Arqueológico Nacional (fig. 6). Se trata de una reproducción del techo de los polícromos, realizado por el profesor Erich Pietsch, del Deutsches Museum, en 1963 e instalado al año siguiente en un recinto excavado a tal fin en los jardines del museo. El sistema utilizado en este caso consiste en la reproducción de las pinturas sobre un soporte modelado a partir de las curvas de nivel del techo de la famosa sala, que habian sido obtenidas por medio de la estereofotogrametría (PIETSCH, 1964). Como señala Delluc (1990), el facsímil de Altamira constituye el único ejemplo de estas carac- 


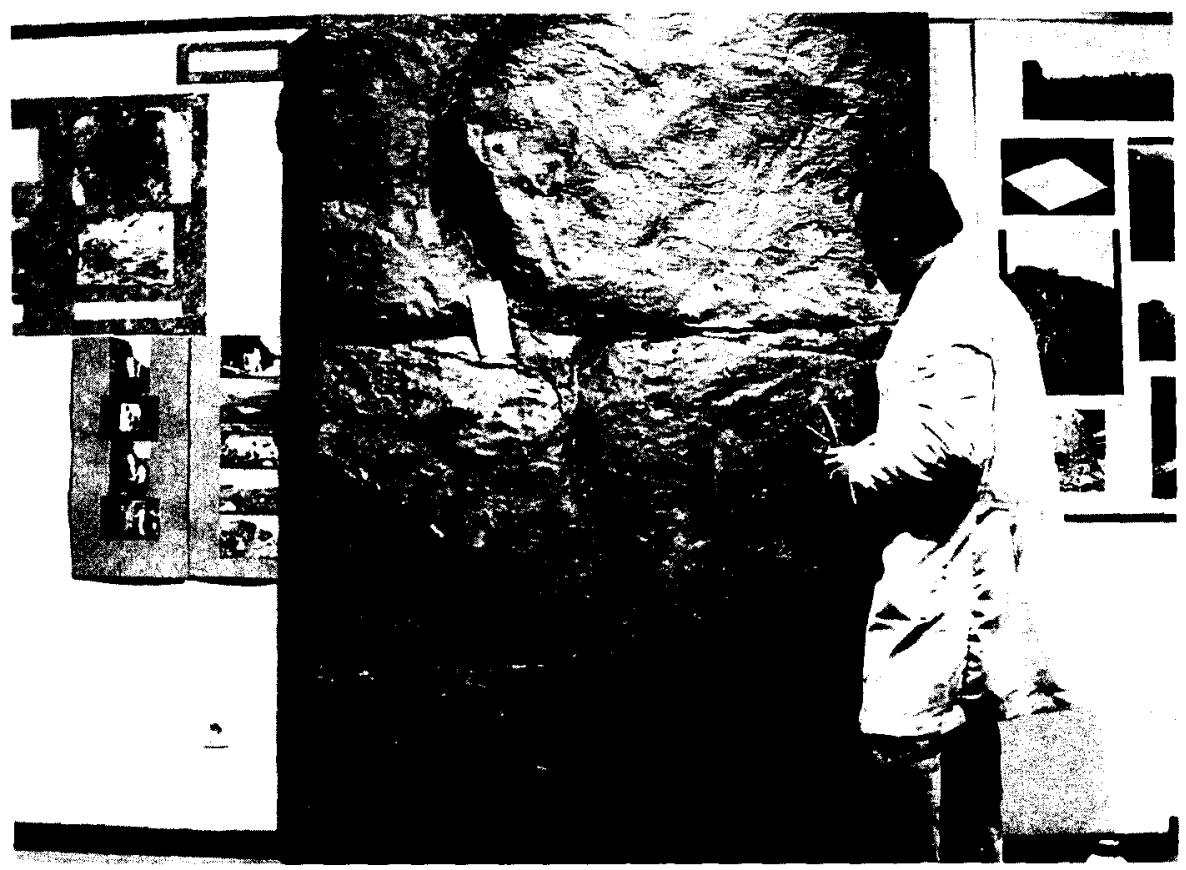

Fig. 5. Instalación del molde de Domingo García en la exposición Arte Rupestre de las Comunidades de Castilla y León y Madrid, U.A.M. 1992 (fotografia facilitada por Alejandro Gallego Fernández).

terísticas realizado en el mundo junto con el más reciente de Lascaux II (Dordoña, Francia).

Ya se ha hecho referencia anteriormente a la presencia de originales en el Museo Arqueológico de Barcelona. Se trata concretamente de tres fragmentos del abrigo de Calapatá, arrancados por Juan Cabré hacia 1905, y que en la actualidad se exponen, en bastante mal estado de conservación, en el interior de una vitrina bajo una luz tenue.

En algunas ocasiones se incluye material arqueológico obtenido en prospecciones o excavaciones de las mismas estaciones artísticas, que puede estar relacionado con el proceso de elaboración del arte (pintaderas canarias en el Museo Arqueológico Nacional y pigmentos prehistóricos en el Arqueológico de Barcelona).

4. Fecha de instalación: En los pocos casos en que se detalla este aspecto, se evidencia una relativa antigüedad en las instalaciones. En el proceso de remodelación que actualmente están experimentando buena 


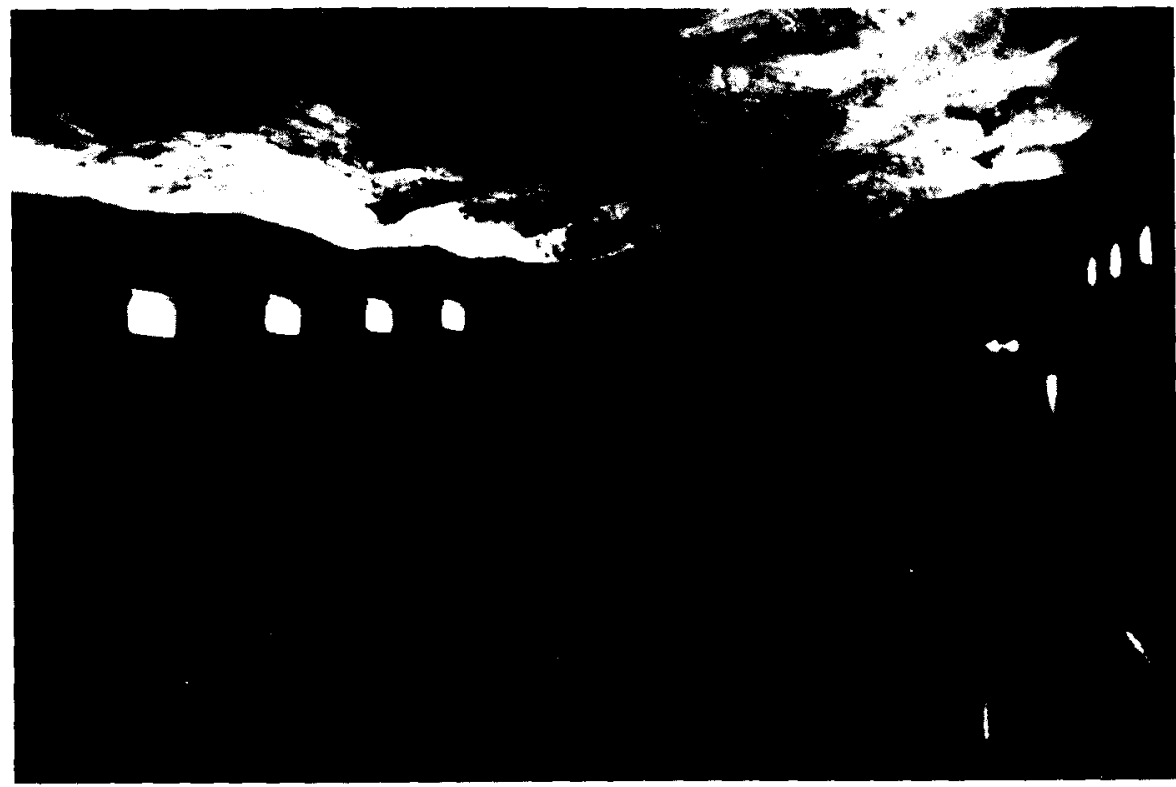

Fig. 6. Vista general del facsímil de Altamira en el M.A.N.

parte de los museos españoles se está procediendo a la incorporación de algunos detalles, sin que se abandonen totalmente los dispositivos antiguos. Entre otros ejemplos, se puede señalar el Museo de Murcia, cuyo montaje, fechado en los años 60 , se ha visto completado con diversas fotografias en color durante la remodelación llevada a cabo entre 1989-1993. En el Museo de Segovia se está acometiendo la renovación total del edificio, mientras que en Pontevedra está prevista la transformación de varias de las salas, incluyendo la dedicada a arte rupestre.

5. Material complementario: Entendiendo por tal el que no forma parte de la exposición propiamente dicha, y se refiere total o parcialmente al arte rupestre. Se puede encontrar en el propio museo (gabinete didáctico, sala de audiovisuales, biblioteca, etc.) o bien a la venta.

Desafortunadamente, la interpretación a esta pregunta del cuestionario no fue la adecuada en todos los casos, por lo que no se cuenta con información detallada al respecto. De cualquier forma, parece que este tipo de material no se circunscribe específicamente al tema del arte rupestre, sino que es de carácter mucho más general.

Destaca la iniciativa del gabinete didáctico del Museo Arqueológico de Barcelona, en colaboración con la ONCE, consistente en la elabora- 
ción de un dossier de arte rupestre para invidentes que incluye una serie de láminas en las que se reproduce, en positivo, la silueta de algunos de los motivos existentes en las estaciones artísticas.

Entre los objetos a la venta destacan la colección de reproducciones enteladas y diapositivas (diez) del Techo de los Polícromos de Altamira a la venta en el Museo Arqueológico Nacional, elaborados por la Editorial Sílex, y el video sobre arte rupestre del Museo de Cuenca, con un apartado especial dedicado a la estación de Villar del Humo. También es frecuente la presencia en las tiendas de los museos de algunas de las numerosas publicaciones especializadas sobre el tema.

6. Valoración de las instalaciones actuales: En general, los conservadores están poco satisfechos con la situación actual, para la que no prevén grandes posibilidades de cambio.

La mayoría de las quejas manifestadas se refieren a la escasa dotación económica de los museos, inadecuación de los medios, antigũedad de los montajes y, por último, a la poca flexibilidad del espacio expositivo, problemas que, por otra parte, no son exclusivos del tema que nos ocupa.

7. Justificación del arte rupestre en los museos: Era ésta una de las preguntas más interesantes del cuestionario, ya que daba opción a los especialistas a manifestar sus opiniones y sugerencias personales.

Las respuestas obtenidas evidencian una total defensa de la museización del arte rupestre "in situ" y, consecuentemente, una escasa valoración de su presencia en el museo tradicionamente entendido. En este último caso, el arte se consideraría siempre un elemento complementario para los distintos períodos culturales, es decir, un auxiliar del discurso expositivo. Sin duda, el aspecto que más pesa a la hora de emitir esta opinión es la dificultad de exposición en sala inherente al carácter inmueble de estos testimonios.

De cualquier manera, es cierto que la mayoría de los profesionales consultados reconocieron tener pocas posibilidades de centrar su atención en este asunto concreto, ante la existencia de otros problemas más acuciantes, referentes incluso a la propia subsistencia de la institución.

No se puede acabar este apartado sin hacer mención de las razones expuestas para justificar la ausencia de arte rupestre en un número considerable de museos $(38,5 \%$ sobre el total de las respuestas), que se pueden resumir en dos: a) inexistencia de manifestaciones artísticas en el entorno geográfico del museo y b) rechazo de estas manifestaciones, bien sea por las dificultades de contextualización (problemática crono- 
lógico-interpretativa), bien por considerar que su exposición carece de interés.

\section{VALORACIÓN CRITTICA Y SUGERENCIAS}

La idea principal que se desprende del análisis de los datos obtenidos es la poca valoración por parte de los especialistas del arte como objeto museable.

Esta situación encontraría su explicación fundamentalmente en los siguientes factores:

a) Problemas inherentes al proceso de museización del arte rupestre, derivados de su carácter inmueble y de la problemática cronológico-interpretativa.

b) Escasa formación museográfica, sobre todo por lo que se refiere a las nuevas tendencias, de una parte de los profesionales que trabajan en estas instituciones.

c) Más recientemente, la proliferación de proyectos de Parques Culturales con arte rupestre, considerada por parte de los especialistas la manera más idónea de presentar el arte, ha contribuido a que se desdeñe el museo tradicional como marco de exposición y didáctica.

d) Desde un punto de vista más general, las dificultades económicas y de gestión que actualmente atraviesan muchos museos han influido en la paralización de buena parte de los procesos de remodelación y renovación de las salas.

Toda estas causas provocan la no inclusión del arte en muchos montajes de salas de Prehistoria (tal y como atestiguan los datos de la encuesta, una buena parte de los museos que han participado en este estudio no exponen ningún ejemplo de estas manifestaciones), o el mantenimiento de montajes antiguos. En este segundo caso se comprueba cómo se sigue recurriendo a los sistemas clásicos de reproducción (calcos, dibujos, fotografías) ${ }^{8}$, infravalorando las enormes posibilidades de medios como el vídeo, los montajes de diapositivas o la informática (que en un futuro no muy lejano permitiría, por ejemplo, la reconstrucción del

a Estos sistemas han permanecido prácticamente inalterados desde principios de siglo, momento en el que precisamente se estructura la base teórico-metodológica por la que se ha regido esta disciplina científica hasta momentos muy recientes. 


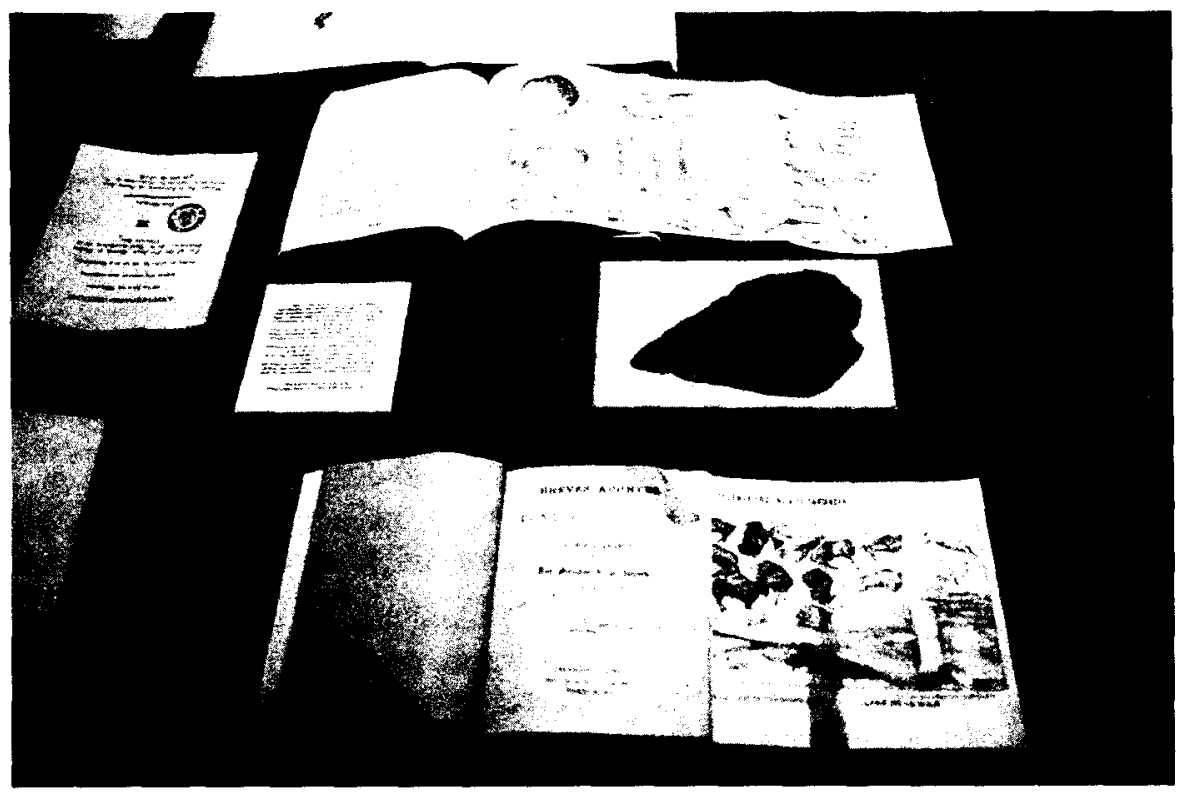

Fig. 7. Vitrina del M.A.N. con el folleto de Marcelino Sanz de Sautuola (1880), primera publicación de arte rupestre en España.

proceso artístico o la "visita" detallada de las estaciones mediante programas de realidad virtual), etc.

Hay que destacar que, a pesar de su antigüedad, algunos de los materiales expuestos o conservados en los almacenes no se deben despreciar, pues constituyen bienes patrimoniales en sí mismos (por ejemplo, la documentación de campañas antiguas, que en muchas ocasiones permanece aún inédita) (fig. 7). Es el caso del Museo de Pontevedra, que custodia los moldes y calcos de la colección Sobrino Buhigas. En general, su recuperación se está propiciando en toda Europa, a través de organismos culturales de la Comunidad y de la propia Unesco.

La escasa preocupación por «modernizar" los museos arqueológicos empieza a superarse en diversos países europeos donde se aboga por la utilización de criterios más didácticos y dinámicos (sin olvidar las cuestiones de marketing), partiendo de una postura que implica mayor compromiso con la sociedad a la que se sirve (DAMM, 1987).

Como en tantas otras ocasiones, esta tendencia aún no tiene su reflejo en los museos españoles, poco abiertos a las innovaciones, tanto en lo que se refiere a la renovación de las disposiciones ya existentes, como a la creación de nuevos montajes. 


\section{PROPUESTA DE EXPOSICIÓN}

Como resumen y complemento de todo lo dicho, se detallan a continuación las consideraciones o sugerencias que, a nuestro juicio, deberían presidir cualquier proceso de museización de arte rupestre, proceso que se verá muy influido por la existencia o no de estas manifestaciones en el entorno geográfico del museo (provincial o regional).

1. Integrar el arte con la cultura material correspondiente; en el caso de que posea entidad suficiente, se puede considerar la opción de dedicarle un espacio específico.

2. Incluir ejemplos de los distintos ciclos artísticos existentes en la región, al margen de criterios estéticos.

3. Presentar mapas de dispersión de las estaciones artísticas a nivel provincial y/o regional. Resulta muy útil la inclusión en estos mismos mapas de algunos de los principales yacimientos de los períodos correspondientes.

4. Recurso a la fotografía en color acompañada de dibujos y croquis de lectura. La escala más conveniente es la 1:1, desaconsejándose las tintas planas y las reconstrucciones excesivas.

Los montajes fotográficos, audiovisuales y moldes resultan muy ilustrativos; estos últimos siempre serán realizados por especialistas, siguiendo las tẻcnicas más contrastadas e inocuas para los conjuntos artísticos.

Todos estos sistemas serán incluidos atendiendo a las medidas de conservación más adecuadas.

En el caso de estaciones muy significativas y emblemáticas, cuya apertura pública represente graves peligros para su conservación, se puede plantear la conveniencia de realizar un facsímil (en cualquier caso, lo más próximo posible al encalve artístico) ${ }^{9}$. A la hora de emprender empresas de esta envergadura, resulta casi imprescindible recurrir al patrocinio privado, que tan positivos resultados ha dado en el caso de Lascaux (Delluc, 1990; Herreros, C., 1983b).

5. En cuanto a la redacción de textos y elaboración de paneles explicativos, sería interesante partir de una encuesta previa a los visitantes del museo, con una doble finalidad: concretar los conocimientos y per-

- En el momento de finalizar este artículo (mayo de 1994) se ha hecho público el proyecto de construir un gran museo de arte prehistórico en Altamira y una réplica de la cueva, con capacidad para recibir unos 250.000 visitantes al año. 


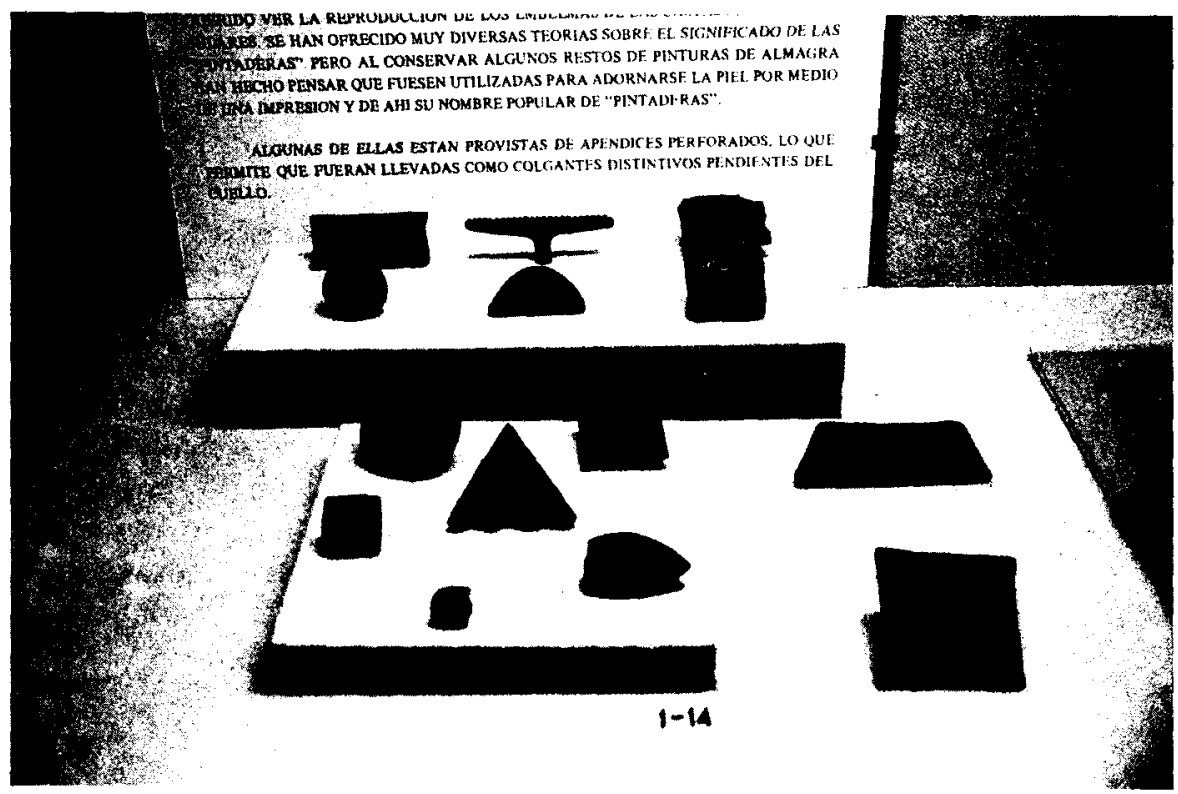

Fig. 8. Pintaderas en la vitrina sobre arte rupestre canario del M.A.N.

cepciones erróneas acerca de la Prehistoria en general, y, sobre todo, conocer sus expectativas respecto a la exposición.

A partir de los datos de la encuesta se elaborarían los textos, mínimos en extensión, que podrian incluir datos sobre cronología, interpretación e identificación de los motivos, con una terminología accesible a un público no especializado.

6. Puede ser interesante informar sobre las técnicas de realización de las manifestaciones artísticas, mediante la exposición de distintos elementos como lámparas, pigmentos, pinceles, punzones, etc. (originales o reproducciones) e incluso reconstrucciones ideales del proceso artístico (fig. 8). El recurso al paralelo etnográfico debe estar siempre presidido por la máxima cautela.

En el caso de que el museo o sus colaboradores hayan participado en trabajos de reproducción de las estaciones artísticas expuestas, se puede incluir información al respecto.

7. El arte rupestre ofrece buenas posibilidades para el desarrollo de iniciativas en el gabinete didáctico, desde las conocidas hojas didácticas hasta numerosas actividades en las áreas de participación (por ejemplo, animando a los niños a realizar sus propias pinturas sobre una super- 
ficie rocosa adaptada a tal fin en la que puedan experimentar con diferentes pigmentos y familiarizarse con los motivos más comunes).

Existen algunas publicaciones de divulgación, adaptadas a los distintos niveles educativos, que también podrian proporcionarse en el museo ${ }^{10}$.

8. En cuanto al material complementario a la venta, son imprescindibles las diapositivas, postales y guías (si se trata de conjuntos muy importantes, pueden ser más específicas y si no el tema puede constituir un apartado en la guía del museo). También se pueden elaborar folletos temáticos complementarios. La presencia de publicaciones científicas, opcional en muchos casos, es inexcusable en los lugares donde el arte tenga especial relevancia.

9. El museo puede servir para fomentar la visita a los enclaves con arte rupestre que estén preparados a tal fin; en este caso, deberá ofrecer material de apoyo para la visita de las estaciones artísticas, creando en el público la necesaria concienciación social acerca del valor y fragilidad de estos testimonios.

10. Un caso aparte lo constituyen los museos de regiones en las que no existen esos testimonios prehistóricos, circunstancia que apenas se da en nuestro país por la enorme difusión geográfica de los distintos ciclos artísticos. El recurso a las exposiciones itinerantes parece la solución más adecuada en este caso concreto.

Como resumen de todo lo expuesto, conviene subrayar que el arte rupestre es un objeto museable, con todas sus limitaciones y posibilidades, que deben ser conocidas por los responsables encargados de su exposición. La idea que debe presidir su inclusión en el museo debe ser la perfecta coherencia con la línea argumental del discurso expositivo, para lo que se pueden utilizar gran variedad de dispositivos museográficos.

Hay que recordar que la justificación última para incluir el arte en los museos, además de su valor como documento arqueológico, es su gran poder evocador, reconocido por antropólogos y artistas.

\section{BIBLIOGRAFIA}

Balldellou, V. (1990): “El parque cultural del Rio Vero (Huesca)", Jornadas sobre Parques con Arte Rupestre. D.G.A., págs. 149 y ss.

10 Por ejemplo, el libro de John Gilbert (1981): La vida de los hombres en la prehistoria. Ediciones Altea. Para la explicación del arte rupestre en las aulas, véase Anna Alonso TEJADA (1987): Art préhistoric: taller escola. Manual per els profesors. Barcelona. Sobre otras iniciativas, véase BATTAGLIOL et al., 1985. 
Balldellou, V., et al. (1988): “Algunas reflexiones sobre los Parques Culturales y el Arte Rupestrem, B.A.E.A.R., 1. Barcelona.

BATtAGLIOU, M.; BoIt, M.; SChVoerer, M. (1985): "Archéologie expérimentale en milieu scolaire", Actes de la première rencontre sur sensibilisation des enfants à l'existence, l'étude et la sauvegarde du patrimoine culturel archéologique. Segunda parte. PACT News, 16, págs. 18-27.

Collado Villalba, O. (1992): Parque cultural de Albarracín. Gobierno de Aragón.

DAMM, Annette (1987): "La reinterpretación de la edad de piedra en Moesgard", Museum 154 , págs. $107-112$.

Delluc, Y Gilles Brigitte (1990): "Lascaux II, une reussite touristique pour le Périgord", Les dossiers d'Historie et d'Archéologie 152 (septiembre), págs. 78-80.

Generalitat Valenciana (1985): Parque cultural de la Valltorta: Anteproyecto y cartografía. Castellón de la Plana.

Gómez Barrera, J.A.; Jimeno Martinez, A.; Fernandez Moreno, J.J.; Yusta Bonilla, J.P.: «Propuesta del Monte Valonsadero como parque arqueológico", // Symposium de arqueología soriana. Homenaje a D. Teógenes Ortego y Frías 19-21 octubre 1989, vol. II. Publicaciones de la Excelentísima Diputación Provincial de Soria. Colección Temas Sorianos 20, págs. 1145-1166.

HALL, M. (1987): On Display. Londres.

HerRero VILLA, Santiago (c. 1983a): "Altamira, Estudios sobre el estado actual", Revista de Arqueología, núm. 6, págs. 22-31.

- (c. 1983b): "Cueva de Lascaux. 40 años de trabajo por su supervivencia", Revista de Arqueologia, núm. 10, págs. 30-39.

- Jornadas sobre parques con arte rupestre. Diputación General de Aragón, Departamento de Cultura y Educación. Zaragoza 1989.

MARTIN DE GuZMAN, Celso, et al. (1993): «El parque arqueológico de la Cueva Pintada de Galdar (Gran Canaria)", Seminario de Parques Arqueológicos, Madrid 13-15 de diciembre de 1989. Madrid, M.C., págs. 23-43.

PIETSCH, E. (1964): Altamira y la prehistoria de la tecnologia química. Patronato de Investigación Científica y Técnica "Juan de la Cierva". C.S.J.C. Madrid.

\section{ANEXO 1}

\section{CUESTIONARIO SOBRE EL TRATAMIENTO MUSEOGRÁFICO DEL ARTE RUPESTRE}

1. ¿El Museo expone algún ejemplo de Arte Rupestre en sus salas?

SI/NO (en caso negativo pase a la pregunta núm. 15).

2. Enumere las estaciones artísticas expuestas.

3. Justifique, si es posible, el criterio de selección adoptado.

4. En caso de existir Arte Rupestre en la localidad, ¿se expone alguna reproducción del mismo en el Museo?

5. Las reproducciones expuestas se localizan:

- en las salas específicas

- en las salas de los períodos correspondientes

- otros.

6. Tipo de reproducciones expuestas: 


\section{Calcos}

Dibujos

Fotografias en $b / n$

Fotografías en color

Moldes

Otros

¿Se hace uso de croquis de lectura?

7. ¿Podria especificar aproximadamente cuándo y con qué motivo fueron realizadas dichas reproducciones?

8. En términos generales, ¿considera adecuado el sistema de reproducción elegido?

9. ¿Cuál es, a su modo de ver, la forma más adecuada para exponer el Arte Rupestre en un Museo?

10. ¿La exposición del Arte Rupestre se ilustra con algún resto de cultura material? ¿Cuál?

11. ¿Existe material ilustrativo a disposición del público (libros, postales, videos, otros)?

12. En términos generales, ¿está satisfecho del tratamiento que el Arte Rupestre recibe en el Museo?

13. ¿Qué modificaciones haria si pudiera?

14. Añada cualquier otro comentario que considere de interés.

\section{ANEXO 2 \\ INSTITUCIONES COLABORADORAS}

ANDALUCIA:

Museo de Almeria.

Museo Arqueológico Provincial de Córdoba (*).

Museo de Málaga.

Museo Arqueológico Provincial de Sevilla.

ARAGON:

Museo de Huesca (*).

BALEARES:

(*) Museos que exponen Arte Rupestre en sus salas. 
Museo Arqueológico de Ibiza.

Museo de Menorca.

Castilla-la Mancha:

Museo de Cuenca (*).

Castilla y León:

Museo de Burgos.

Museo de León ( ${ }^{\star}$ ).

Museo de Salamanca (*).

Museo de Segovia (*).

Museo Numantino de Soria (*).

Museo Arqueológico Provincial de Valladolid (*).

Cataluña:

Museo Arqueológico de Barcelona (*).

Museo de Gerona (*).

Museo Nacional Arqueológico de Tarragona.

Museo de Olérdola ( ${ }^{\star}$ ).

\section{EXTREMADURA:}

Museo Arqueológico Provincial de Badajoz (*).

GaLICIA:

Museo Provincia de Orense (*).

Museo de Pontevedra (*).

MAORID:

Museo Arqueológico Nacional (*) ${ }^{11}$.

MURCIA:

Museo de Murcia (*).

Navarra:

Museo de Navarra.

LA RIOJA:

Museo de La Rioja.

VALENCIA:

Museo de Valencia ( $\left.{ }^{\star}\right)$.

11 Todos los comentarios relativos a la instalación del Museo Arqueológico Nacional son responsabilidad exclusiva de las autoras, al haber declinado la conservadora de prehistoria realizar una valoración de los mismos. 